\title{
Young age increases the risk of lymph node positivity but improves prognosis in patients with bladder cancer treated via cystectomy: a population-based study
}

\author{
Tianming Ma ${ }^{1,2}$, Zijian Tian ${ }^{1,2}$, Lingfeng Meng ${ }^{1,2}$, Wei Zhang ${ }^{1}$, Jiawen Wang ${ }^{1}$, Xiaodong Liu ${ }^{1,2}$, \\ Xiaonan Wang ${ }^{2,3}$, Yaoguang Zhang ${ }^{1,2}$ \\ ${ }^{1}$ Department of Urology, Beijing Hospital, National Center of Gerontology, Institute of Geriatric Medicine, Chinese Academy of Medical Sciences, \\ Beijing, China; ${ }^{2}$ Graduate School of Peking Union Medical College, Chinese Academy of Medical Sciences, Beijing, China; ${ }^{3}$ Department of \\ Radiology, Beijing Hospital, National Center of Gerontology, Institute of Geriatric Medicine, Chinese Academy of Medical Sciences, Beijing, China \\ Contributions: (I) Conception and design: All authors; (II) Administrative support: Y Zhang; (III) Provision of study materials or patients: T Ma, Z \\ Tian, X Wang, Y Zhang; (IV) Collection and assembly of data: T Ma, L Meng, W Zhang, J Wang, X Liu; (V) Data analysis and interpretation: T \\ Ma, Z Tian, X Wang; (VI) Manuscript writing: All authors; (VII) Final approval of manuscript: All authors. \\ Correspondence to: Yaoguang Zhang. Department of Urology, Beijing Hospital, National Center of Gerontology, Institute of Geriatric Medicine, \\ Chinese Academy of Medical Sciences, No. 1 DaHua Road, Dong Dan, Beijing 100730, China. Email: zhang003887@sina.com.
}

\begin{abstract}
Background: Age and lymph node positivity are significant prognostic indicators in patients with bladder cancer. This study aimed to investigate the impact of age on lymph node positivity and bladder cancer outcomes.

Methods: Patients with bladder cancer who underwent cystectomy with at least one lymph node examined between 2004 and 2015 were identified from the Surveillance, Epidemiology, and End Results database. Cochran-Armitage trend tests and logistic regression analyses were used to evaluate the association between age and lymph node positivity in all $\mathrm{T}$ stages. Multivariate Cox regression analysis was used to analyze the effect of age on overall survival (OS) and cancer-specific survival (CSS).

Results: Overall, 13,251 patients were identified, 648 of whom were under 50 years of age (4.89\%). Lymph node positivity was negatively associated with increasing age in each stage except in non-invasive-muscular bladder cancer. In the multivariable analysis, age was an independent prognostic factor for OS and CSS in both the overall cohort and the lymph node positivity group.

Conclusions: In patients with bladder cancer undergoing cystectomy, young age at diagnosis is associated with a higher risk of lymph node positivity and superior outcomes. These findings may guide clinicians in selecting suitable treatments, determining the aggressiveness of lymph node involvement, and predicting survival outcomes in patients of different ages.
\end{abstract}

Keywords: Bladder cancer (BCa); age at diagnosis; lymph node positivity; SEER; prognosis

Submitted Feb 01, 2021. Accepted for publication Jul 27, 2021.

doi: 10.21037/tau-21-99

View this article at: https://dx.doi.org/10.21037/tau-21-99

\section{Introduction}

Bladder cancer (BCa) is among the most common types of cancer, with high mortality and steadily rising morbidity worldwide (1). Recent data have also indicated that $\mathrm{BCa}$ represents the neoplasm with the fourth highest incidence and eighth highest mortality among American men in
2021 (2). Radical cystectomy (RC) combined with lymph node dissection (LND) is recommended as the standard treatment for muscle-invasive bladder cancer (MIBC), although the treatment can also be applied in patients with high-risk and/or recurrent non-muscle-invasive tumors $(3,4)$. Previous studies have reported that as many as $25 \%$ 
of patients with RC have lymph node (LN) metastases, and that increases in the number of affected LNs are closely related to the progression of the pathologic stage $(5,6)$. Additional research has indicated that $\mathrm{LN}$ involvement is a vital indicator of worse prognosis after RC with LND, which is independently associated with recurrence and cancer-specific mortality $(7,8)$.

Several studies have revealed that age can affect LN positivity $(\mathrm{LN}+)$ in patients with rectal cancer, thyroid cancer, and various other malignant tumors (9-11). A recent study demonstrated that young patients with MIBC were more likely to have LN metastases than older patients (12). Other studies have also reported a significant association between age and $\mathrm{LN}$ status in patients treated with RC $(13,14)$. However, given the limitations of these studies, the precise association between $\mathrm{LN}+$ and patient age at diagnosis of $\mathrm{BCa}$ remains to be determined.

Although the effect of age on $\mathrm{LN}+$ remains unclear, researchers tend to agree that young patients treated with $\mathrm{RC}$ are more likely to experience a favorable prognosis than their older counterparts $(15,16)$. However, given that $\mathrm{LN}_{+}$ itself is associated with poor prognosis, this speculation remains controversial. Therefore, the present study aimed to investigate the effect of age on $\mathrm{LN}+$ and survival outcomes in patients who underwent cystectomy using data from the Surveillance, Epidemiology, and End Results (SEER) database. We present the following article in accordance with the STROBE reporting checklist (available at https://dx.doi.org/10.21037/tau-21-99).

\section{Methods}

\section{Study population}

The SEER database collects data on cancer morbidity, survival, and mortality, encompassing approximately $28 \%$ of the US population. In this study, we analyzed SEER data for adult patients ( $\geq 18$ years old) diagnosed with BCa [International Classification of Disease for Oncology (ICD-O) site code C67.0-67.9] from 2004 to 2015. The diagnosis of $\mathrm{BCa}$ was confirmed via pathologic examination, and 16,167 patients who underwent surgery following the examination of at least one $\mathrm{LN}$ were initially included. Patients who had not undergone cystectomy (RX Summ-Surg Prim Site codes 50, 60-64, 70-74, 80, $\mathrm{n}=1,532)$ and those without complete clinicopathological data $(n=105)$ were excluded. To eliminate the effect of preoperative radiation on $\mathrm{LN}$ collection and positivity, patients who received radiotherapy prior to surgery were excluded. Patients with distant metastasis or whose bladder malignancy was not their first primary cancer were also excluded. Finally, 13,251 patients were included in the overall cohort (Figure 1).

\section{Statistical analysis}

Age was considered a categorical variable, and patients were classified into 10-year age groups. Considering the relatively small number of patients under the age of 50 years, we classified them all into one group, so patients were divided into " $<50$ ", "50-59", "60-69", "70-79", “ $\geq 80$ " groups according to their age at diagnosis. Year of $\mathrm{BCa}$ diagnosis was stratified into the following three categories: "2004-2007", "2008-2011", and "2012-2015". Histology was classified as "pure urothelial carcinoma (UC)" or "nonurothelial variant histology (VH)", the latter of which was further divided into squamous cell, neuroendocrine, adenocarcinoma, and other VH. T stage was regrouped into non-muscle-invasive bladder cancer (NMIBC: Ta + Tis + T1) and stages T2, T3, and T4. Chi-square and KruskalWallis $\mathrm{H}$ tests with Bonferroni's post hoc tests were used to compare clinicopathological features among different age groups. The number of lymph nodes examined (LNE) was compared among the age groups stratified by $\mathrm{T}$ stage using the Kruskal-Wallis $\mathrm{H}$ test. Cochran-Armitage trend tests were used to evaluate trends in $\mathrm{LN}+$ with age among the $\mathrm{T}$ stage groups. We conducted univariate and multivariate logistic regression analyses for $\mathrm{T}$ stage considering $\mathrm{LN}+$ as the outcome. Age at diagnosis, sex, race, marital status, year of diagnosis, tumor grade, histology, and the number of LNE were included as covariates. Results are represented as odds ratios (ORs) and 95\% confidence intervals (CIs) with age $\geq 80$ years as the reference group. Overall survival (OS) and cancer-specific survival (CSS) were compared using Kaplan-Meier curves with the log-rank test, stratified by age groups. Hazard ratios (HRs) and 95\% CIs were determined using multivariable Cox proportional hazards models to evaluate the effect of age on OS and CSS in subgroups according to $\mathrm{T}$ stage. The adjusted covariates were as follows: sex, race, marital status, year of diagnosis, tumor grade, histology, LN status (LNs positive vs. LNs negative), the number of LNE, and chemotherapy. Furthermore, subgroup analyses were performed in patients with at least one positive LN. All analyses were performed using SPSS v. 25 and $\mathrm{R}$ v.4.0.3. All tests were two-sided, and $\mathrm{P}<0.05$ was considered statistically significant. 


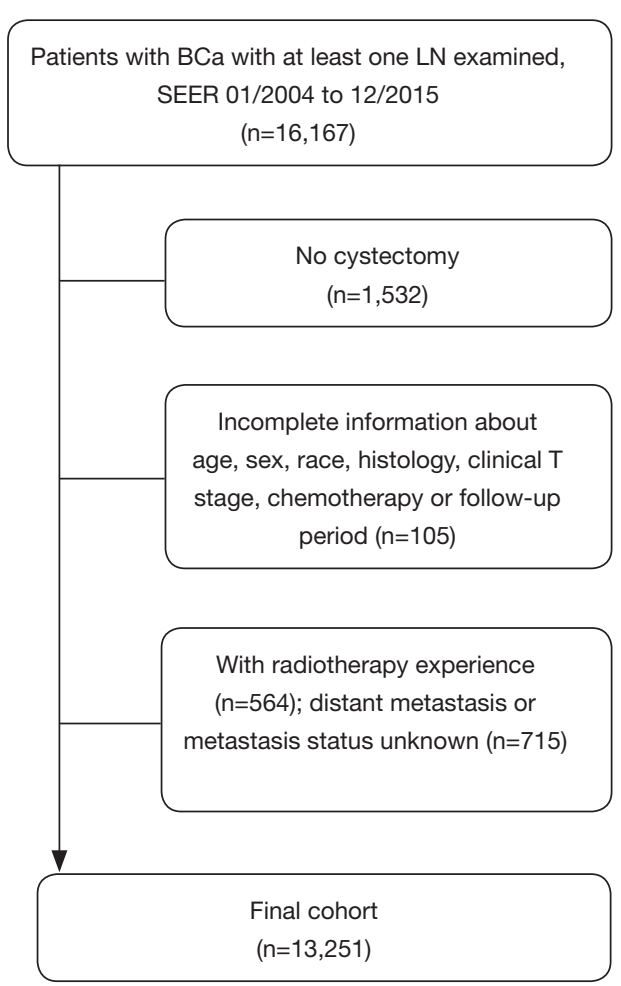

Figure 1 Study cohort selection. BCa, bladder cancer; LN, lymph node; SEER, Surveillance, Epidemiology, and End Results.

\section{Ethics statement}

The study was conducted in accordance with the Declaration of Helsinki (as revised in 2013). As data from the SEER program is available for the public and does not need patient informed consent, and ethical approval was waived by the local Ethics Committee of Beijing Hospital.

\section{Results}

\section{Baseline characteristics of the overall cohort}

A total of 13,251 patients were identified in our study. Table 1 shows the demographic and clinicopathological characteristics of the included patients. Generally, the majority of patients were men $(n=10,081,76.08 \%)$ and Caucasian ( $\mathrm{n}=11,775,88.86 \%)$. Patients aged $70-79$ years accounted for the greatest proportion $(n=4,359,32.90 \%)$. Only 648 patients $(4.89 \%)$ were aged $<50$ years, and the remaining patients fell into the following age groups: $50-59$ years $(n=2,247,16.96 \%), 60-69$ years $(n=4,274$, $32.25 \%)$, and $\geq 80$ years $(\mathrm{n}=1,723,13.00 \%)$, respectively.
The most frequently encountered grade and $\mathrm{T}$ stage were grade IV $(n=8,388,63.30 \%)$ and stage T2 $(n=5,187$, $39.14 \%)$. Most patients had pure UC ( $\mathrm{n}=12,458,94.02 \%)$, while VH was confirmed in only 793 patients (5.98\%). Patients aged $\geq 80$ years received chemotherapy less frequently than those aged $<50$ years $(18.28 \%$ vs. $51.08 \%$, $\mathrm{P}<0.001)$. The number of $\mathrm{LNE}$ was highest in patients aged 50-59 years with NMIBC or T2 disease and in those aged $<50$ years with T3 disease $(n=17)$. In contrast, the median number of LNE was lowest in the $\geq 80$ group, regardless of T stage. The average numbers of LNE were $14,14,13$, and 13 for NMIBC, T2, T3, and T4, respectively (Table 2).

\section{Effect of age at diagnosis on $L N+$}

We grouped patients according to $\mathrm{T}$ stage to evaluate the impact of age on $\mathrm{LN}+$. In the cohort, 3,228 patients (24.36\%) had positive LNs. The rates of $\mathrm{LN}+$ were $3.95 \%$, $12.59 \%, 36.03 \%$, and $46.48 \%$ for patients with NMIBC, $\mathrm{T} 2$, T3, and T4 disease, respectively. The rate of $\mathrm{LN}+$ was negatively associated with age in each stage, except for the NMIBC stage (Table 3). We then performed multivariate logistic regression analyses to further identify the correlation between age at diagnosis and LN+. Sex, race, marital status, year of diagnosis, tumor grade, histology, and the number of LNE were used as covariates of the adjusted model. Our findings indicated that age was independently associated with $\mathrm{LN}+$ in the $\mathrm{T} 2-\mathrm{T} 4$ stages $(\mathrm{P}<0.05$, Table 4). Patients aged $<50$ years had a higher risk of $\mathrm{LN}+$ than the reference group (aged $\geq 80$ years) in stages T2 and T4 (adjusted OR: 1.771; 95\% CI: 1.129-2.777; $\mathrm{P}=0.003$ ), (adjusted OR: 1.660; 95\% CI: 1.039-2.653; $\mathrm{P}=0.001$ ), respectively. Patients with stage T3 disease in the 50-59 years group had the highest probability of $\mathrm{LN}+$ (adjusted OR: 1.313; 95\% CI: $1.055-1.635 ; \mathrm{P}<0.001$ ). However, age was not significantly associated with $\mathrm{LN}+$ in the NMIBC group $(\mathrm{P}=0.142)$.

\section{Survival analysis}

Multivariate Cox regression analysis indicated that younger age was significantly correlated with improved OS and CSS in all stages (all $\mathrm{P}<0.05$, Table 5), relative to the reference group (aged $\geq 80$ years), except for the CSS of patients aged 70-79 years with stage T2 disease (HR: 0.867; 95\% CI: 0.711-1.057; $\mathrm{P}=0.158)$. Patients aged $<50$ years had the highest OS and CSS rates when compared with other age groups (Figure 2). 
Table 1 Demographic and clinicopathological characteristics by age at diagnosis

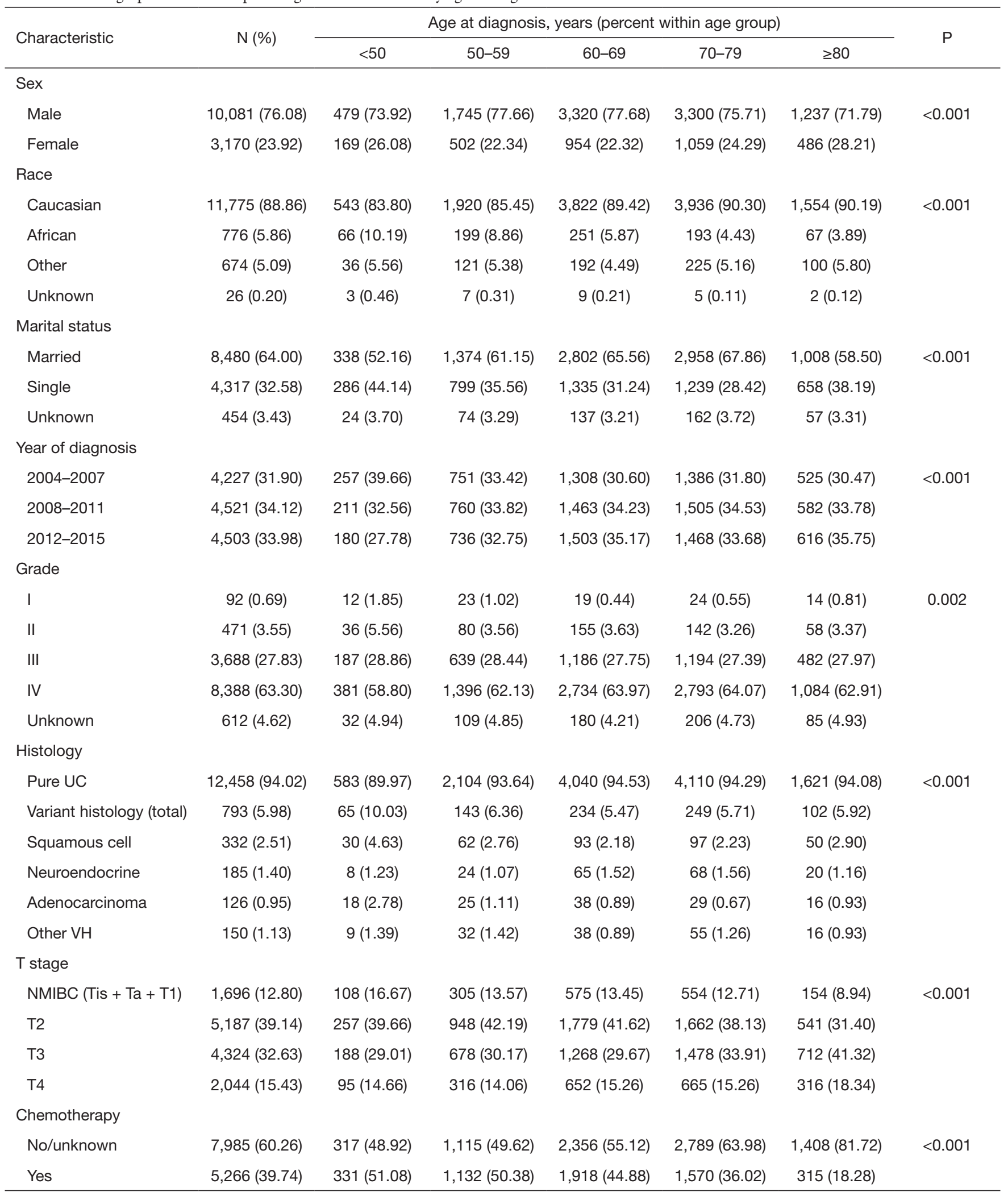

UC, urothelial carcinoma; VH, variant histology; NMIBC, non-muscle-invasive bladder cancer. 
Table 2 Number of LNE stratified by age and T stage

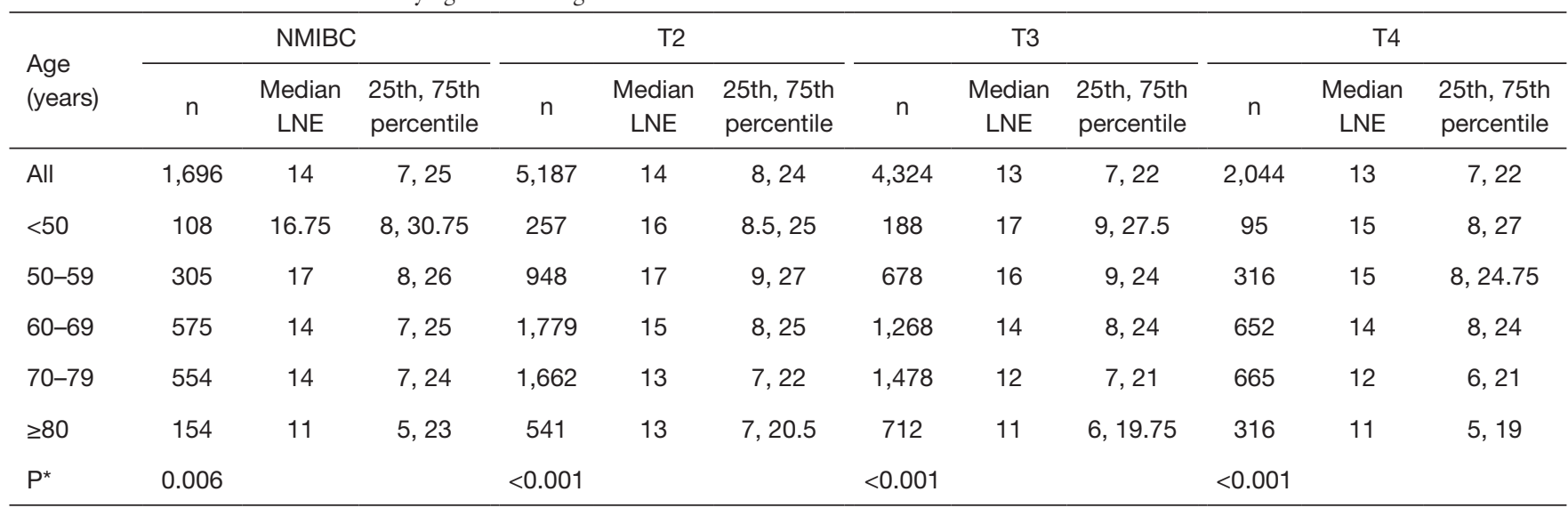

*, from Kruskal-Wallis $\mathrm{H}$ test. LNE, lymph nodes examined; NMIBC, non-muscle-invasive bladder

Table $3 \mathrm{LN}+$ rate and age within T stage groups

\begin{tabular}{|c|c|c|c|c|c|c|c|c|}
\hline $\begin{array}{l}\text { Age } \\
\text { (years) }\end{array}$ & \multicolumn{2}{|c|}{ NMIBC } & \multicolumn{2}{|c|}{$\mathrm{T} 2$} & \multicolumn{2}{|c|}{ T3 } & \multicolumn{2}{|c|}{$\mathrm{T} 4$} \\
\hline All & 1,696 & 67 (3.95) & 5,187 & 653 (12.59) & 4,324 & 1,558 (36.03) & 2,044 & $950(46.48)$ \\
\hline$<50$ & 108 & $9(8.33)$ & 257 & $40(15.56)$ & 188 & $68(36.17)$ & 95 & 53 (55.79) \\
\hline $50-59$ & 305 & 14 (4.59) & 948 & $141(14.87)$ & 678 & 291 (42.92) & 316 & 165 (52.22) \\
\hline $70-79$ & 554 & 21 (3.79) & 1,662 & $192(11.55)$ & 1,478 & 458 (30.99) & 665 & 291 (43.76) \\
\hline$\geq 80$ & 154 & $3(1.95)$ & 541 & $49(9.06)$ & 712 & $253(35.53)$ & 316 & $132(41.77)$ \\
\hline $\mathrm{P}^{*}$ & 0.11 & & $<0.01$ & & $<0.01$ & & 0.02 & \\
\hline
\end{tabular}

*, from Cochran-Armitage trend test. LN+, lymph node positivity; NMIBC, non-muscle-invasive bladder cancer.

\section{Subgroup analysis}

Among patients with $\mathrm{LN}_{+}$, those under 50 years of age accounted for only $5.27 \%$ of patients. Sex $(\mathrm{P}=0.137)$, year of diagnosis $(\mathrm{P}=0.053)$, and tumor grade $(\mathrm{P}=0.053)$ did not significantly differ between the groups. All demographic and clinicopathological characteristics are summarized in Table S1. The Kaplan-Meier curves show that patients aged $<50$ years still exhibited the longest overall and cancer-specific median survival times (Figure 3). In the multivariable Cox regression analysis, patients aged $<70$ years also had a better prognosis, but there was no significant risk of decreased OS (HR: 0.896; 95\% CI: 0.790-1.917; $\mathrm{P}=0.090$ ) or CSS (HR: 0.890; 95\% CI: $0.766-$ $1.035 ; \mathrm{P}=0.129)$ in patients aged $70-79$ years relative to the reference group (Table S2).

\section{Discussion}

Tumor stage and LN status are considered the most significant factors affecting the oncological outcomes of patients undergoing RC and LND. Multiple factors including tumor stage, grade, and histology are used to predict the risk of $\mathrm{LN}$ metastasis after RC $(17,18)$. In the present study, we analyzed the predictive value of age for $\mathrm{LN}+$ and prognosis in patients with BCa. Our findings indicated that, although young patients were more likely to exhibit LN+ during stages T2-T4, their OS and CSS remained higher than those in other groups despite $\mathrm{T}$ stage. Moreover, young age remained a predictor of better prognosis in the subgroup of patients with $\mathrm{LN}$ metastasis.

Previous studies have reported the influence of age on $\mathrm{LN}+$ in $\mathrm{BCa}$. In their analysis of 15,624 patients 


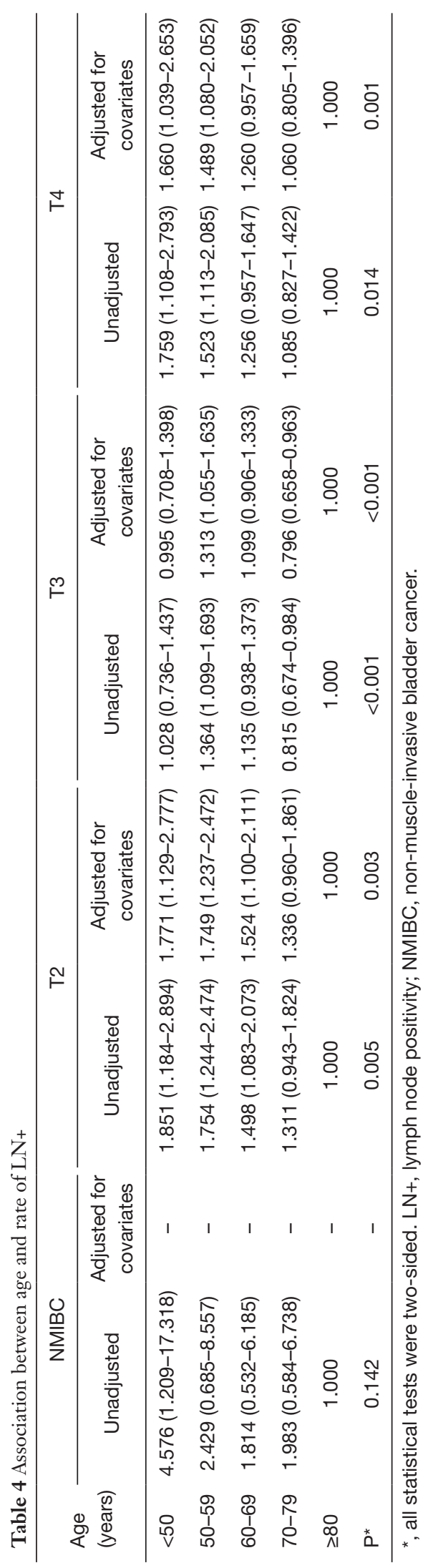

with MIBC, Tian et al. demonstrated that age was an independent risk factor for $\mathrm{LN}_{+}$, and that the rate of $\mathrm{LN}+$ decreased as the age of diagnosis increased despite $\mathrm{T}$ stage (12). Although these results are consistent with the findings of the present study, the previous study did not include patients with NMIBC who had undergone RC. In addition, the authors did not report tumor histology, OS, or CSS. Hellenthal et al. reported that the probability of LND (at least one node) after cystectomy in patients with BCa decreased by approximately $20 \%$ with every 10-year increase in age from 1988 to 2004 after adjusting for other covariates (14). Koppie et al. observed similar results, noting that age influenced the total number of LNE after RC (19). Nevertheless, both studies only focused on the LNE while ignoring the impact of age on $\mathrm{LN}+$. In their analysis of data from patients with UC undergoing RC, Almassi et al. observed that LNE increased (7 to 24), while $\mathrm{LN}+$ decreased (19\% to 8.7\%), from 1995 to 2015 (20). However, they did not group patients by age for further discussion. Additionally, in our study, patient age was not significantly associated with $\mathrm{LN}+$ in the NMIBC group. We suspect that the less aggressive nature of the tumor and unknown treatment strategies (e.g., adjuvant intravesical chemotherapy prior to RC) during the NMIBC stage resulted in the inclusion of only 67 (3.95\%) patients with NMIBC who experienced LN metastasis following RC. This limited sample size undoubtedly affected outcomes in our analysis of the relationship between age and $\mathrm{LN}+$.

Biological differences between young and old patients with BCa may be related to the impact of age on $\mathrm{LN}+$. Several studies have demonstrated that $\mathrm{VH}$ is significantly associated with the likelihood of LN metastasis $(18,21)$. In our study, younger patients exhibited higher rates of $\mathrm{VH}$ in both the entire cohort $(10.03 \%$ for age $<50$ vs. $5.92 \%$ for age $\geq 80)$ and $\mathrm{LN}+$ group $(14.71 \%$ for age $<50$ vs. $7.09 \%$ for age $\geq 80$ ). Findings from a recent study also support the notion that young patients with $\mathrm{VH}$ tend to exhibit more locally aggressive features after RC (22). Moreover, young patients are more likely to have a low frequency of chromosome 9 deletions and FGFR3 mutations, whereas TP53 and ERBB2 mutations occur frequently in old patients and have been associated with the invasiveness of BCa $(23,24)$. Notably, Migaldi et al. demonstrated that low cyclinD1 expression and a high MIB-1 index are correlated with an increased risk of recurrence in patients $<45$ years of age (25). Furthermore, aging contributes to numerous changes in the immune system, and older adults are more susceptible to cancer or infection due to decreased 
Table 5 Association between age and survival outcomes in subgroups according to T stage

\begin{tabular}{|c|c|c|c|c|c|}
\hline T stage subgroups & Age at diagnosis & \multicolumn{2}{|c|}{ Overall survival } & \multicolumn{2}{|c|}{ Cancer-specific survival } \\
\hline \multirow[t]{4}{*}{ NMIBC } & $<50$ & $0.187(0.120-0.293)$ & $<0.001$ & $0.326(0.171-0.622)$ & 0.001 \\
\hline & $50-59$ & $0.213(0.155-0.292)$ & $<0.001$ & $0.367(0.225-0.596)$ & $<0.001$ \\
\hline & $60-69$ & $0.286(0.221-0.370)$ & $<0.001$ & $0.396(0.258-0.608)$ & $<0.001$ \\
\hline & $\geq 80$ & 1.000 (reference) & & 1.000 (reference) & \\
\hline \multirow[t]{3}{*}{ T2 } & $<50$ & $0.213(0.160-0.283)$ & $<0.001$ & $0.392(0.274-0.560)$ & $<0.001$ \\
\hline & $50-59$ & $0.366(0.311-0.431)$ & $<0.001$ & $0.564(0.449-0.708)$ & $<0.001$ \\
\hline & $60-69$ & $0.470(0.410-0.540)$ & $<0.001$ & $0.610(0.498-0.747)$ & $<0.001$ \\
\hline \multirow[t]{5}{*}{ T3 } & $<50$ & $0.566(0.460-0.698)$ & 0.027 & $0.777(0.613-0.984)$ & 0.036 \\
\hline & $50-59$ & $0.591(0.516-0.676)$ & $<0.001$ & $0.728(0.618-0.856)$ & $<0.001$ \\
\hline & $60-69$ & $0.645(0.576-0.722)$ & $<0.001$ & $0.719(0.624-0.828)$ & $<0.001$ \\
\hline & $70-79$ & $0.817(0.735-0.909)$ & 0.001 & $0.861(0.752-0.986)$ & 0.030 \\
\hline & $\geq 80$ & 1.000 (reference) & & 1.000 (reference) & \\
\hline \multirow[t]{3}{*}{ T4 } & $<50$ & $0.543(0.410-0.719)$ & $<0.001$ & $0.660(0.477-0.913)$ & 0.012 \\
\hline & $50-59$ & $0.635(0.529-0.761)$ & $<0.001$ & $0.748(0.602-0.929)$ & 0.009 \\
\hline & $60-69$ & $0.617(0.528-0.720)$ & $<0.001$ & $0.671(0.555-0.812)$ & $<0.001$ \\
\hline
\end{tabular}

$\mathrm{HR}$, hazard ratio; $\mathrm{Cl}$, confidence interval; NMIBC, non-muscle-invasive bladder cancer.

immunity $(26,27)$. Recent studies have highlighted that aging can affect degeneration of the cortex and medulla in the LNs, modify the response of LN stromal cells to challenge, and reduce lymphatic flow to nodes (26-28). These age-related variations may account for the effect of age on $\mathrm{LN}+$.

In the present study, we noticed that young age was a predictor of better survival outcomes in both the total population and the $\mathrm{LN}+$ cohort. Although this result is consistent with some previous findings $(15,16,22,29)$, it is contradictory to others $(30,31)$. We speculate that different methods for grouping patients based on age at diagnosis may have caused these discrepancies. In general, younger patients tend to be healthier, with favorable immune function and nutritional status as well as a lower risk of comorbidities and fewer treatment- related complications. Previous studies have suggested that younger patients prefer to undergo more complete, radical, and combined treatment modalities than older patients (e.g., LND or extensive LND during RC combined with chemotherapy) - a finding also reflected in our study (13-15,19-29). Indeed, in our study, rates of chemotherapy and the median number of LNE were higher in patients $<50$ years of age than in patients $\geq 80$ years of age. These findings indicate that young patients may be more inclined to receive and better tolerate aggressive multimodality treatments than their older counterparts. Furthermore, the seemingly contradictory finding that young patients exhibit good prognosis despite a high incidence of $\mathrm{LN}_{+}$ may be associated with age-dependent differences in biology, immunological surveillance, and BCa host factors. In addition, socioeconomic status, delayed diagnosis and/or 

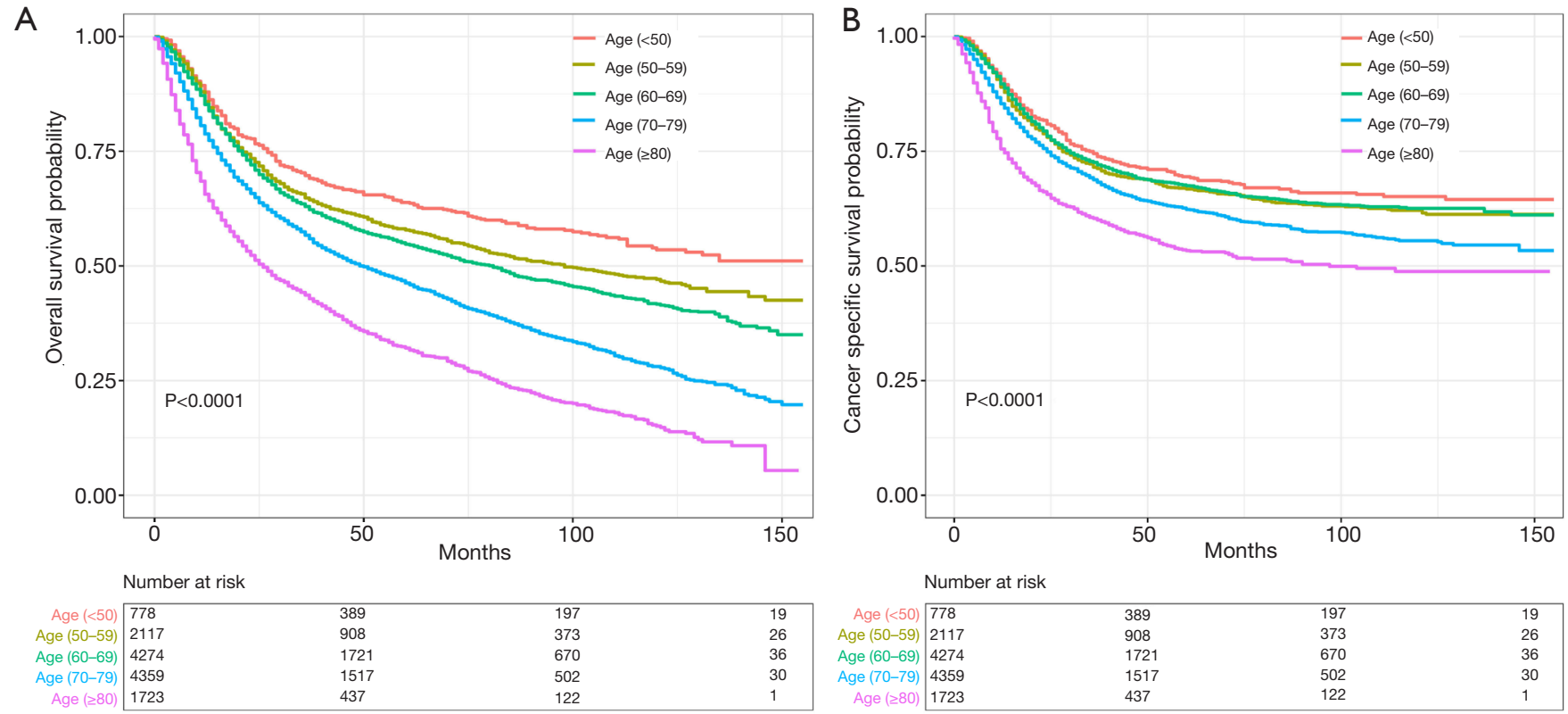

Figure 2 Kaplan-Meier survival curves of entire cohort stratified by age at diagnosis. (A) Overall survival; (B) cancer-specific survival.

A
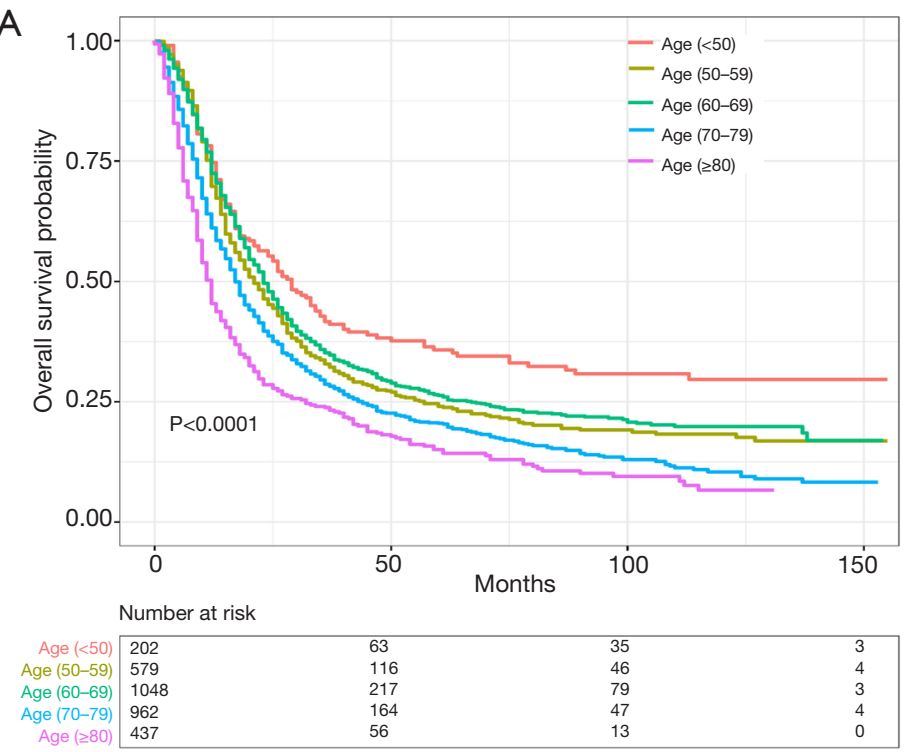

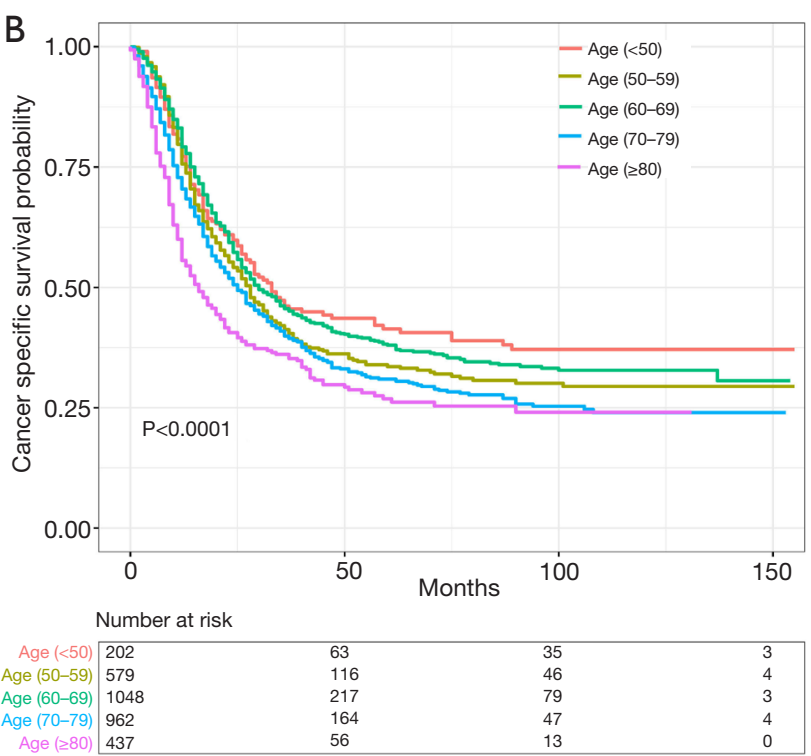

Figure 3 Kaplan-Meier survival curves of lymph node positive cohort stratified by age at diagnosis. (A) Overall survival; (B) cancer-specific survival.

treatment, and the physicians' tendency to perform radical treatment may also influence survival among older adults. Therefore, further studies are required to address these issues.

Accurate prediction of $\mathrm{LN}$ involvement is vital, as it can impact treatment strategies for patients with $\mathrm{BCa}$, especially for those who are ready to undergo RC to assess the need for and extent of LND. At present, whether LND can improve oncological outcomes and the appropriate extent of LND remain controversial. A recent systematic 
review of 23 studies concluded that any kind of LND has advantages over no LND (5). Nevertheless, Larcher et al. found that, when compared with $\mathrm{RC}$ alone, $\mathrm{RC}$ with pelvic LND is associated with improved outcomes in younger and healthier patients, but not in older and sicker patients (32). In addition, immediate RC and LND may be considered overtreatment for patients with high-risk NMIBC, such as $\mathrm{T} 1$ high-grade $\mathrm{BCa}$, due to their potentially lower risk of metastasis and progression (4). Based on their systematic review, Klaassen et al. recommended RC with LND to improve staging and prognosis in patients diagnosed with T1 high-grade BCa (33), given that approximately $5-25 \%$ of patients may have occult $\mathrm{LN}$ metastases at the time of cystectomy $(5,6,34)$. To date, numerous studies have suggested that extended LND provides a survival benefit in patients undergoing $\mathrm{RC}(5,13,19)$. Bi et al. conducted a systematic review and meta-analysis, noting that extended LND is associated with improved recurrence-free survival (RFS), in contrast to non-extended LND. Their subgroup analysis revealed that extended LND was associated with better RFS for patients with p T3-4 BCa regardless of LN status (35). Bruins et al. also demonstrated that extended LND was superior to lesser degrees of dissection, whereas super-extended LND was not associated with further benefits (5). However, Choi et al. reported that there were no significant differences in the estimated 5-year RFS, CSS, or OS rates among patients undergoing standard LND, extended LND, or super-extended LND (36). Recently, a prospective randomized trial reported that extended LND failed to offer noteworthy superiority over limited LND with respect to the RFS, CSS, and OS of patients with BCa (37). Notably, no differences in major complication rates after 30 and 90 days were reported in the limited and extended LND groups, consistent with the findings of another previous study (38). Therefore, larger randomized prospective trials are necessary to explore the proper extent of LND to benefit patients with BCa. Although older age at RC appears safe, with survival benefits that may outweigh the risk of perioperative mortality and morbidity, our study and previous investigations have demonstrated that older patients are less likely to undergo this aggressive procedure, especially LND $(15,29,39)$. As the proper extent and benefits of LND remain controversial, while the incidence of $\mathrm{LN}+$ is low in older patients, active assessment of lymph node involvement and other factors is necessary for providing individualized treatment to older patients.

The present study had several limitations, including its retrospective design. In addition, although we considered factors such as $\mathrm{VH}$, year of diagnosis, and other clinicopathological variables in our multivariate analysis, young patients may have received more aggressive clinical treatment than older patients, resulting in systematic bias. Furthermore, some important data (e.g., lymphovascular invasion, tumor size and location, adjuvant intravesical therapy, and chemotherapy type) were not available in this study. For example, two categories ("No/Unknown" or "Yes") were assigned to chemotherapy, which may lead to information bias and influence HR of variables. Noncancer-related factors also represented the primary cause of patient death, leading to a much greater difference between OS and CSS. However, we could not further explore the impact of age on OS in the present study, because we were unable to assess the frailty status and comorbidities among patients. In addition, despite excluding patients with radiotherapy experience, there were still some patients who underwent neoadjuvant chemotherapy and/or salvage RC after trimodality therapy, which may have influenced LN+ and prognosis. Finally, the data were derived only from the population covered by the SEER database, and our findings may not be generalizable to other geographic locations.

\section{Conclusions}

Our analysis demonstrates that there is an increased risk of $\mathrm{LN}+$ and a greater likelihood of better prognosis in young patients with $\mathrm{BCa}$ treated via cystectomy, after adjusting for other predictive factors. These findings may guide clinicians in selecting suitable treatments, determining the aggressiveness of LN involvement, and predicting survival outcomes in patients of different ages. Further studies are required to verify our findings and to assess factors predictive of $\mathrm{LN}+$ in patients with high-risk NMIBC across age.

\section{Acknowledgments}

We would like to thank Editage (www.editage.cn) for English language editing.

Funding: This study was supported by the National Key Research and Development Program of China (grant number: 2018YFC2002202).

\section{Footnote}

Reporting Checklist: The authors have completed the STROBE reporting checklist. Available at https://dx.doi. 
org/10.21037/tau-21-99

Conflicts of Interest: All authors have completed the ICMJE uniform disclosure form (available at https://dx.doi. org/10.21037/tau-21-99). The authors have no conflicts of interest to declare.

Ethical Statement: The authors are accountable for all aspects of the work in ensuring that questions related to the accuracy or integrity of any part of the work are appropriately investigated and resolved. The study was conducted in accordance with the Declaration of Helsinki (as revised in 2013). As data from the SEER program is available for the public and does not need patient informed consent, and ethical approval was waived by the local Ethics Committee of Beijing Hospital.

Open Access Statement: This is an Open Access article distributed in accordance with the Creative Commons Attribution-NonCommercial-NoDerivs 4.0 International License (CC BY-NC-ND 4.0), which permits the noncommercial replication and distribution of the article with the strict proviso that no changes or edits are made and the original work is properly cited (including links to both the formal publication through the relevant DOI and the license). See: https://creativecommons.org/licenses/by-nc-nd/4.0/.

\section{References}

1. Sung H, Ferlay J, Siegel RL, et al. Global Cancer Statistics 2020: GLOBOCAN Estimates of Incidence and Mortality Worldwide for 36 Cancers in 185 Countries. CA Cancer J Clin 2021;71:209-49.

2. Siegel RL, Miller KD, Fuchs HE, et al. Cancer Statistics, 2021. CA Cancer J Clin 2021;71:7-33.

3. Stein JP, Lieskovsky G, Cote R, et al. Radical cystectomy in the treatment of invasive bladder cancer: long-term results in 1,054 patients. J Clin Oncol 2001;19:666-75.

4. Babjuk M, Burger M, Compérat EM, et al. European Association of Urology Guidelines on Non-muscleinvasive Bladder Cancer (TaT1 and Carcinoma In Situ) 2019 Update. Eur Urol 2019;76:639-57.

5. Bruins HM, Veskimae E, Hernandez V, et al. The impact of the extent of lymphadenectomy on oncologic outcomes in patients undergoing radical cystectomy for bladder cancer: a systematic review. Eur Urol 2014;66:1065-77.

6. Ou N, Song Y, Liu M, et al. Development and Validation of a Nomogram to Predict Lymph Node Metastasis in
Patients With T1 High-Grade Urothelial Carcinoma of the Bladder. Front Oncol 2020;10:532924.

7. Mari A, Kimura S, Foerster B, et al. A systematic review and meta-analysis of lymphovascular invasion in patients treated with radical cystectomy for bladder cancer. Urol Oncol 2018;36:293-305.

8. Karl A, Carroll PR, Gschwend JE, et al. The impact of lymphadenectomy and lymph node metastasis on the outcomes of radical cystectomy for bladder cancer. Eur Urol 2009;55:826-35.

9. Meyer JE, Cohen SJ, Ruth KJ, et al. Young Age Increases Risk of Lymph Node Positivity in Early-Stage Rectal Cancer. J Natl Cancer Inst 2016;108:djv284.

10. Liu Y, Wang Y, Zhao K, et al. Lymph node metastasis in young and middle-aged papillary thyroid carcinoma patients: a SEER-based cohort study. BMC Cancer 2020;20:181.

11. Jiang Y, Huang W, Xie J, et al. Young age increases risk for lymph node positivity in gastric cancer: A Chinese multiinstitutional database and US SEER database study. J Cancer 2020;11:678-85.

12. Tian Z, Meng L, Wang X, et al. Young age increases the risk of lymph-node metastasis in patients with muscleinvasive bladder urothelial carcinoma. BMC Cancer 2020;20:851.

13. Wang K, Shi H, Mao W, et al. Role of lymph node dissection in radical cystectomy. Oncol Lett 2020;20:409-19.

14. Hellenthal NJ, Ramírez ML, Evans CP, et al. Trends in pelvic lymphadenectomy at the time of radical cystectomy: 1988 to 2004. J Urol 2009;181:2490-5.

15. Chromecki TF, Mauermann J, Cha EK, et al. Multicenter validation of the prognostic value of patient age in patients treated with radical cystectomy. World J Urol 2012;30:753-9.

16. Luzzago S, Knipper S, Palumbo C, et al. Effect of Age on Cancer-specific Mortality in Patients With Urothelial Carcinoma of the Urinary Bladder: A Population-based Competing-risks Analysis Across Disease Stages. Am J Clin Oncol 2020;43:880-8.

17. Kluth LA, Black PC, Bochner BH, et al. Prognostic and Prediction Tools in Bladder Cancer: A Comprehensive Review of the Literature. Eur Urol 2015;68:238-53.

18. Rice KR, Koch MO, Kao CS, et al. Lymph node metastases in patients with urothelial carcinoma variants: influence of the specific variant on nodal histology. Urol Oncol 2015;33:20.e23-9.

19. Koppie TM, Vickers AJ, Vora K, et al. Standardization of 
pelvic lymphadenectomy performed at radical cystectomy: can we establish a minimum number of lymph nodes that should be removed? Cancer 2006;107:2368-74.

20. Almassi N, Cha EK, Vertosick EA, et al. Trends in Management and Outcomes among Patients with Urothelial Carcinoma Undergoing Radical Cystectomy from 1995 to 2015: The Memorial Sloan Kettering Experience. J Urol 2020;204:677-84.

21. Guo L, Zhang L, Wang J, et al. Pelvic Lymph Node Dissection During Cystectomy for Patients With Bladder Carcinoma With Variant Histology: Does Histologic Type Matter? Front Oncol 2020;10:545921.

22. de la Calle CM, Washington SL 3rd, Lonergan PE, et al. Bladder cancer in patients younger than 40 years: outcomes from the National Cancer Database. World J Urol 2021;39:1911-6.

23. Weyerer V, Schneckenpointner R, Filbeck T, et al. Immunohistochemical and molecular characterizations in urothelial carcinoma of bladder in patients less than 45 years. J Cancer 2017;8:323-31.

24. Hedegaard J, Lamy P, Nordentoft I, et al. Comprehensive Transcriptional Analysis of Early-Stage Urothelial Carcinoma. Cancer Cell 2016;30:27-42.

25. Migaldi M, Rossi G, Maiorana A, et al. Superficial papillary urothelial carcinomas in young and elderly patients: a comparative study. BJU Int 2004;94:311-6.

26. Hadamitzky C, Spohr H, Debertin AS, et al. Agedependent histoarchitectural changes in human lymph nodes: an underestimated process with clinical relevance? J Anat 2010;216:556-62.

27. Masters AR, Hall A, Bartley JM, et al. Assessment of Lymph Node Stromal Cells as an Underlying Factor in Age-Related Immune Impairment. J Gerontol A Biol Sci Med Sci 2019;74:1734-43.

28. Pan WR, Suami H, Taylor GI. Senile changes in human lymph nodes. Lymphat Res Biol 2008;6:77-83.

29. Goossens-Laan CA, Leliveld AM, Verhoeven RH, et al. Effects of age and comorbidity on treatment and survival of patients with muscle-invasive bladder cancer. Int J

Cite this article as: Ma T, Tian Z, Meng L, Zhang W, Wang J, Liu X, Wang X, Zhang Y. Young age increases the risk of lymph node positivity but improves prognosis in patients with bladder cancer treated via cystectomy: a population-based study. Transl Androl Urol 2021;10(8):3375-3385. doi: 10.21037/tau-21-99
Cancer 2014;135:905-12.

30. Horovitz D, Turker P, Bostrom PJ, et al. Does patient age affect survival after radical cystectomy? BJU Int 2012;110:E486-93.

31. Janisch F, Yu H, Vetterlein MW, et al. Do Younger Patients with Muscle-Invasive Bladder Cancer have Better Outcomes? J Clin Med 2019;8:1459.

32. Larcher A, Sun M, Schiffmann J, et al. Differential effect on survival of pelvic lymph node dissection at radical cystectomy for muscle invasive bladder cancer. Eur J Surg Oncol 2015;41:353-60.

33. Klaassen Z, Kamat AM, Kassouf W, et al. Treatment Strategy for Newly Diagnosed T1 High-grade Bladder Urothelial Carcinoma: New Insights and Updated Recommendations. Eur Urol 2018;74:597-608.

34. Fritsche HM, Burger M, Svatek RS, et al. Characteristics and outcomes of patients with clinical T1 grade 3 urothelial carcinoma treated with radical cystectomy: results from an international cohort. Eur Urol 2010;57:300-9.

35. Bi L, Huang H, Fan X, et al. Extended vs non-extended pelvic lymph node dissection and their influence on recurrence-free survival in patients undergoing radical cystectomy for bladder cancer: a systematic review and meta-analysis of comparative studies. BJU Int 2014;113:E39-48.

36. Choi SY, You D, Hong B, et al. Impact of lymph node dissection in radical cystectomy for bladder cancer: How many vs how far? Surg Oncol 2019;30:109-16.

37. Gschwend JE, Heck MM, Lehmann J, et al. Extended Versus Limited Lymph Node Dissection in Bladder Cancer Patients Undergoing Radical Cystectomy: Survival Results from a Prospective, Randomized Trial. Eur Urol 2019;75:604-11.

38. Brössner C, Pycha A, Toth A, et al. Does extended lymphadenectomy increase the morbidity of radical cystectomy? BJU Int 2004;93:64-6.

39. Montie JE. Radical cystectomy in the elderly: comparison of survival between younger and older patients. J Urol 2005;174:1254-5. 
Supplementary

Table S1 Demographic and clinicopathological characteristics by age at diagnosis in LN+ group

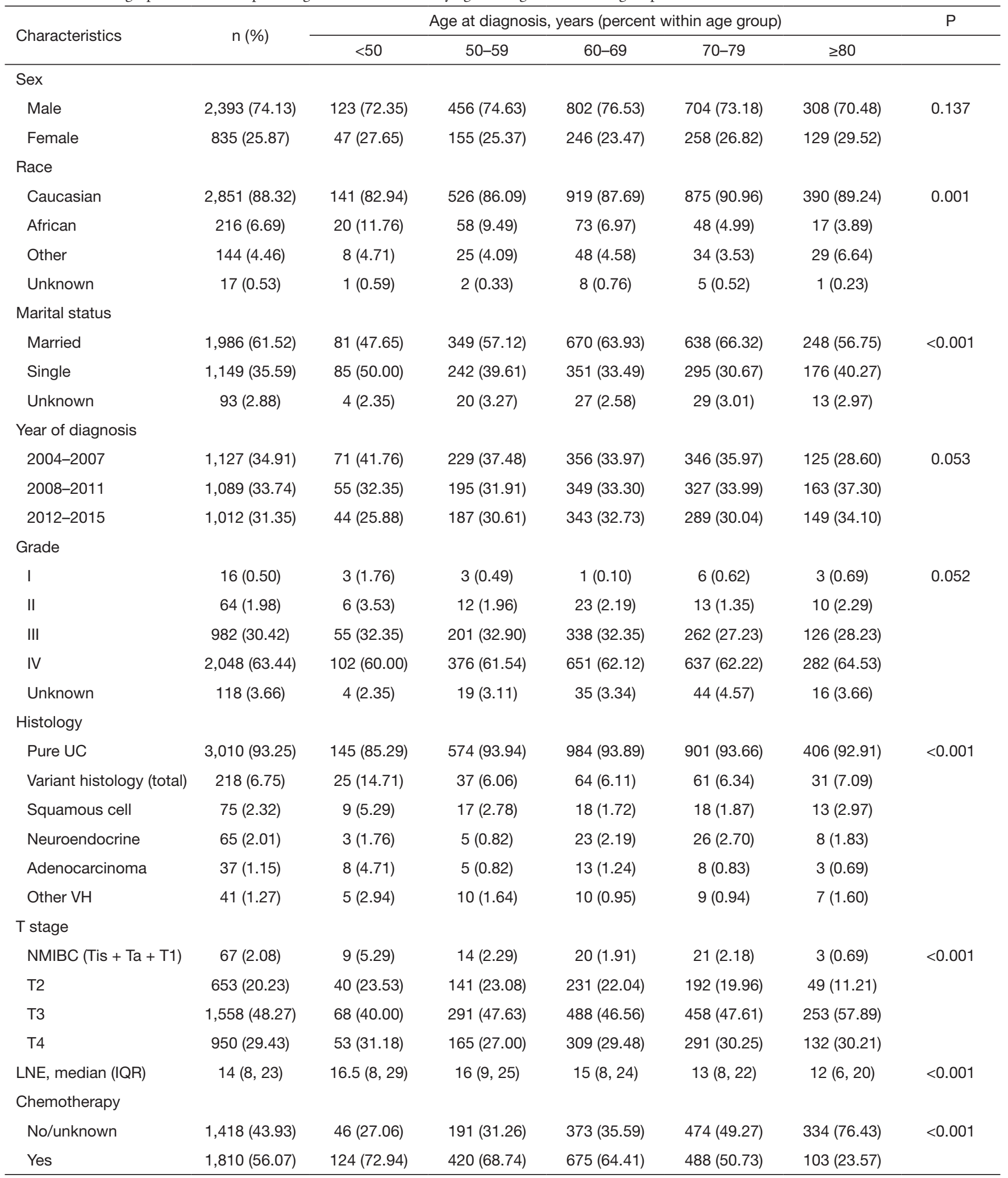

LN+, Iymph node positivity; UC, urothelial carcinoma; VH, variant histology; NMIBC, non-muscle-invasive bladder cancer; IQR, interquartile range. 
Table S2 Association of age and survival outcomes in subgroups according to T stage in LN+ group

\begin{tabular}{|c|c|c|c|c|}
\hline Age at diagnosis & \multicolumn{2}{|c|}{ Overall survival } & \multicolumn{2}{|c|}{ Cancer specific survival } \\
\hline$<50$ & $0.643(0.519-0.798)$ & $<0.001$ & $0.754(0.593-0.960)$ & 0.022 \\
\hline $50-59$ & $0.771(0.668-0.890)$ & $<0.001$ & $0.820(0.693-0.969)$ & $<0.020$ \\
\hline $60-69$ & $0.723(0.636-0.823)$ & $<0.001$ & $0.731(0.628-0.852)$ & $<0.001$ \\
\hline$\geq 80$ & 1.000 (reference) & & 1.000 (reference) & \\
\hline
\end{tabular}

$\mathrm{LN}+$, lymph node positive; $\mathrm{HR}$, hazard ratio; $\mathrm{Cl}$, confidence interval. 